\title{
The secreted acid trehalase encoded by the CgATH1 gene is involved in Candida glabrata virulence
}

\author{
Rafael G Lopes' ${ }^{1}$ Julián E Muñoz ${ }^{2,3,4}$, Ludmila M Barros', \\ Sergio L Alves-Jr ${ }^{5}$, Carlos P Taborda ${ }^{2,3}$, Boris U Stambuk ${ }^{1 /+}$ \\ ${ }^{1}$ Universidade Federal de Santa Catarina, Centro de Ciências Biológicas, Departamento de Bioquímica, Florianópolis, SC, Brasil \\ ${ }^{2}$ Universidade de São Paulo, Instituto de Ciências Biomédicas, Departamento de Microbiologia, São Paulo, SP, Brasil \\ ${ }^{3}$ Universidade de São Paulo, Faculdade de Medicina, Instituto de Medicina Tropical de São Paulo, Departamento de Dermatologia, \\ Laboratório de Micologia Médica/LIM53, São Paulo, SP, Brasil \\ ${ }^{4}$ Universidad del Rosario, Escuela de Medicina y Ciencias de la Salud, Bogotá, Colombia \\ ${ }^{5}$ Universidade Federal da Fronteira Sul, Laboratório de Bioquímica e Genética, Chapecó, SC, Brasil
}

BACKGROUND Candida glabrata yeast is the second cause of candidiasis worldwide. Differs from other yeasts since assimilates only glucose and trehalose (a characteristic used in rapid identification tests for this pathogen) by secreting into the medium a highly active acid trehalase encoded by the $C g A T H 1$ gene.

OBJECTIVE This study aimed to characterise the function of the acid trehalase in the physiopathology of C. glabrata.

METHODS Gene deletion was performed to obtain a mutant ath $1 \Delta$ strain, and the ability of the ath $1 \Delta$ strain to grow in trehalase, or the presence of trehalase activity in the ath1 $\Delta$ yeast cells, was verified. We also tested the virulence of the ath $1 \Delta$ strain in a murine model of infection.

FINDINGS The ath $1 \Delta$ mutant strain grows normally in the presence of glucose, but loses its ability to grow in trehalose. Due to the high acid trehalase activity present in wild-type cells, the cytoplasmic neutral trehalase activity is only detected in the ath1 $\Delta$ strain. We also observed a significantly lower virulence of the ath1s strain in a murine model of infection with either normal or immunocompromised mice.

MAIN CONCLUSIONS The acid trehalase is involved in the hydrolysis of external trehalose by C. glabrata, and the enzyme also plays a major virulence role during infectivity.

Key words: ATH1 - acid trehalase - neutral trehalase - NTH1 - NTH2 - extracellular trehalose assimilation - virulence - pathogenic yeasts

Fungi are recognised as major agents of nosocomial infections, with emphasis on Candida spp. responsible for the majority of invasive fungal infections. Immunocompromised individuals [due to acquired immune deficiency syndrome (AIDS), diabetes, organ and tissue transplantation, cancer treatments, immune-related diseases, premature birth or advanced age] are at high risk for mucosal or systemic candidiasis. Disseminated candidiasis is a life-threatening disease that remains the most common bloodstream infection in hospitalised patients worldwide. Although the majority of fungal infections in humans are caused by Candida albicans, an increasing number of cases are being attributed to other Candida species, in particular Candida glabrata. ${ }^{(1)}$ Over the last decades $C$. glabrata has emerged as one of the most common and problematic causes of invasive candidiasis, even in Brazil. ${ }^{(2)}$ Systemic infections caused by

doi: 10.1590/0074-02760200401

Financial support: CNPq (process 479812/2006-3, 308627/2015-6,

420480/2018-8, 308389/2019-0), CAPES, FAPESP (process 2016-08730-6).

+ Corresponding author: boris.stambuk@ufsc.br

(1) https://orcid.org/0000-0001-7507-1020

Received 30 July 2020

Accepted 05 October 2020 this yeast have high mortality rates and are difficult to treat due to this yeast's frequent and intrinsic antifungal resistance.(3) This opportunistic pathogen, formerly known as Torulopsis glabrata, can be found as a commensal yeast in healthy individuals, and in the environment mainly in association with mammals, reflecting its commensal lifestyle. Indeed, in some populations (such as diabetics and elderly) $C$. glabrata may be the dominant fungal pathogen.

C. glabrata and C. albicans are quite distinct phylogenetically, suggesting that association of these yeasts with the mammalian host evolved independently in these two pathogens. ${ }^{(4)} C$. glabrata is closely related to Saccharomyces yeasts, and part of the Saccharomycetaceae genus named Nakaseomyces that contains few other pathogenic species. ${ }^{(5)}$ During their evolution, these yeasts underwent a whole-genome duplication (WGD) event, and while the genome of most Saccharomyces yeasts retained or even expanded some gene families to allow, for example, efficient sugar utilisation and fermentation, ${ }^{(6)}$ the $C$. glabrata genome underwent a reductive evolution to streamline its metabolic capacity for its life and success as a commensal pathogen. . $^{(4,5)}$ For example, the loss of the whole pathway to synthesise nicotinic acid, a precursor of $\mathrm{NAD}^{+}$, made this yeast dependent on an external source (e.g., the host) to obtain this vitamin. During the urinary tract infection, the limitation of nicotinic acid is an inducing signal for the expression of the 
EPA genes, a family of adhesins encoded at subtelomeric loci subject to transcriptional silencing mediated by the $\mathrm{NAD}^{+}$-dependent histone deacetylase SIR2.(7) The auxotrophy of C. glabrata for other vitamins (e.g., thiamin and pyridoxine ${ }^{(8)}$ ) has also been used for the biotechnological production of pyruvic acid, and other organic acids, from glucose fermentation by this yeast.

Indeed, as S. cerevisiae and other members of the Saccharomycetaceae clade, C. glabrata is a Crabtreepositive yeast that efficiently ferments glucose even in the presence of oxygen. This species has orthologues of almost all S. cerevisiae genes that participate in fermentation and glycolysis, including 11 hexose transporters and the $S N F 3 / R G T 2$ transceptor genes involved in their regulation. Although the pathogenic C. glabrata presents many components of the cAMP-protein kinase A and glucose repression pathways, these regulatory systems have not been fully characterised. ${ }^{(9)}$ Importantly, while most yeast species in general assimilate and ferment several different sugar substrates (galactose, sucrose, maltose etc.), $C$. glabrata can only assimilate another sugar, the disaccharide trehalose ( $\alpha$-D-glucopyranosyl(1-1)- $\alpha$-D-glucopyranoside). This property was immediately recognised as a cost-effective and rapid means to identify this fungal pathogen ${ }^{(10)}$ facilitating the therapy selection, especially in cases of candidaemia. ${ }^{(3)}$

While in the yeast $S$. cerevisiae two distinct pathways for trehalose assimilation have been described, ${ }^{(1)}$ we have shown that $C$. glabrata consumes and ferments trehalose, with similar parameters to those observed during glucose fermentation, due to the secretion of a highly active acid trehalase (EC 3.2.1.28) into the medium. Furthermore, cloning and heterologous expression of the acid trehalase gene ( $C g A T H 1)$ from $C$. glabrata allowed trehalose fermentation by S. cerevisiae cells. ${ }^{(12)}$ Indeed, the CgATHI acid trehalase was recently confirmed among the 119 proteins secreted by $C$. glabrata into the medium. ${ }^{(13)}$ Aiming to better characterise the function of the secreted acid trehalase, in the present report we have deleted the CgATHI gene from the $C$. glabrata genome and analysed the phenotypic consequences in the mutant strain, including the growth pattern when exposed to glucose or trehalose rich mediums, the trehalase activity, and its virulence in a murine model of infection, either in normal or immunocompromised mice. We also show that the majority of pathogenic/opportunistic Candida yeasts species are able to consume extracellular trehalose, due to the presence of genes encoding acid trehalases in their genomes.

\section{MATERIALS AND METHODS}

Yeast strains, media and growth conditions - Unless otherwise stated, all chemicals used in this research were of analytical grade from Sigma-Aldrich (St. Louis, USA). The C. glabrata strains used in this study are described in the Supplementary data, Table I, and were maintained at $-80^{\circ} \mathrm{C}$. Standard rich YP medium $\left(10 \mathrm{~g} \mathrm{~L}^{-1}\right.$ Difco yeast extract, $20 \mathrm{~g} \mathrm{~L}^{-1}$ Difco Bacto peptone) or minimal YNB medium (6.7 $\mathrm{g} \mathrm{L}^{-1}$ Difco Yeast nitrogen base) were used, supplemented with $20 \mathrm{~g} \mathrm{~L}^{-1}$ of glucose or trehalose or 30 $\mathrm{g} \mathrm{L}^{-1}$ of glycerol. The $\mathrm{pH}$ of the rich medium was adjusted to $\mathrm{pH} 5.0$ with $\mathrm{HCl}$ and, in the case of the minimal YNB medium, $50 \mathrm{mmol} \mathrm{L}^{-1}$ succinate-Tris $\mathrm{pH} 5.0$ was used as buffer. Solid media contained $20 \mathrm{~g} \mathrm{~L}^{-1}$ Difco Bacto agar. Cells were grown aerobically at $28^{\circ} \mathrm{C}$ on a rotary shaker $(160 \mathrm{rpm})$ in cotton-plugged Erlenmeyer flasks filled to $1 / 5$ of the volume with medium and inoculated with 30$40 \mu \mathrm{g}$ cell dry weight $(\mathrm{CDW}) \mathrm{mL}^{-1}$. Alternatively, yeast cells were pre-grown overnight in $3 \mathrm{~mL}$ of YP-20 $\mathrm{g} \mathrm{L}^{-1}$ glucose, and 1:100 dilutions of these pre-cultures were used to inoculate $100 \mu \mathrm{L}$ of rich YP medium containing the indicated sugars in 96-well plates in a Tecan GENios microplate reader (Tecan, Männedorf, Switzerland), to determine the yeast growth at $30^{\circ} \mathrm{C}$. All wells in the plate were tightly sealed with AccuClear Sealing Film (E \& K Scientific, Santa Clara, USA). The growth pattern of each culture was monitored by measuring the $\mathrm{OD}_{600} \mathrm{ev}-$ ery $30 \mathrm{~min}$, with high intensity orbital shaking between measurements. All growth experiments were repeated at least twice, observing that differences between strains were highly reproducible. To perform the murine experiments, the yeasts were subcultured in Sabouraud dextrose broth (Becton, Dickinson and Company, Sparks, USA) at $37^{\circ} \mathrm{C}$ for $24 \mathrm{~h}$ at $150 \mathrm{rpm}$ before each assay.

Strain construction - Standard methods for DNA manipulation and analysis, as well as yeast transformation by the lithium acetate/single-stranded carrier DNA/polyethylene glycol method, were employed.(12) The ORF of the CgATH1 gene (locus CAGLOK05137g) on chromosome K of strain CBS138 (http://cbi.labri.fr/ Genolevures/elt/GAGL) was disrupted by a PCR-based gene replacement procedure ${ }^{(14)}$ Briefly, the $S$. cerevisiae URA3 gene from plasmid YEp24 was amplified with primers PRODIGE-ATH1-F and PRODIGE-ATH1-R (Supplementary data, Table I) using Phusion ${ }^{\circledR}$ HighFidelity DNA Polymerase. The resulting PCR product of $924 \mathrm{bp}$, containing flanking regions (c. $60 \mathrm{nt}$.) of homology to the immediate upstream promoter and immediate downstream region of the $\mathrm{CgATH1}$ gene, was used to transform cells of the competent strain Bg14. After $2 \mathrm{~h}$ of cultivation on YP-20 g L ${ }^{1}$ glucose, the transformed cells were plated onto synthetic complete medium supplemented with $30 \mathrm{~g} \mathrm{~L}^{1}$ of glycerol and incubated at $28^{\circ} \mathrm{C}$. Uracil-positive isolates were tested for proper genomic integration of the $S c U R A 3$ gene at the CgATH1 loci by PCR using a set of three primers: VUATH1-F, VIATH1R or VIURA3-R (Supplementary data, Table I).

Determination of trehalase activity - Trehalose hydrolysis by the periplasmic acid trehalase, and the activity in culture supernatants, was determined as previously described. ${ }^{(12)}$ Total trehalase activity in cell extracts was also determined using $100 \mathrm{mmol} \mathrm{L}^{-1}$ succinate-Hepes

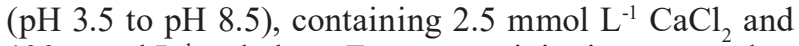
$100 \mathrm{mmol} \mathrm{L}^{-1}$ trehalose. Enzyme activity is expressed as $\mathrm{mU}$, where one unit corresponds to $1 \mu \mathrm{mol}$ of glucose produced $\min ^{-1}$ at $30^{\circ} \mathrm{C}$. Glucose was determined by the glucose oxidase and peroxidase method using a commercial kit (BioDiagnostica-Laborclin, Pinhais, Brazil), and protein was quantified by the Bradford method.

In vivo mice infections and treatments $-\mathrm{BALB} / \mathrm{c}$ isogenic male mice with 6-8 weeks $(\mathrm{n}=5)$ were bred at the University of São Paulo (USP), Brazil, in an animal facility under pathogen-free conditions. Mice were kept in 
cages lined with wood shavings and closed with an autoclaved filter, and served (ad libitum) autoclaved food and water in order to maintain a sterile environment. Cages were changed twice a week in laminar flow hoods. The authors confirm that all procedures involving animals and their care were conducted according to the local ethics committee and international guidelines, approved by the Institutional Animal Care and Use Committee (protocol 042-127-02) of the Institute of Biomedical Sciences, USP.

Disseminated candidiasis in normal mice was induced by caudal intravenous (i.v.) inoculation of $3 \times 10^{5}$ C. glabrata wild-type or ath $1 \Delta$ yeast cells suspended in $100 \mu \mathrm{L}$ of phosphate-buffered saline (PBS) on day 0 . Animals were treated intraperitoneally (i.p.) with PBS (control) or fluconazole $(20 \mathrm{mg} / \mathrm{kg}$ ) every day for a week, starting on day 1. Alternatively, disseminated candidiasis in immunosuppressed animals was performed administrating two doses of $100 \mathrm{mg} / \mathrm{kg}$ cyclophosphamide i.p. four days and one day before infection, and again on day 3 and 7 post-infection, followed by i.v. infection with $1 \times 10^{3}$ C. glabrata wild-type or ath $1 \Delta$ yeast cells suspended in $100 \mu \mathrm{L}$ of PBS on day 0 . Animals were sacrificed eight days after infection, kidneys and spleen were removed and weighed and tissues were individually homogenised by mechanical disruption in $1 \mathrm{~mL}$ of PBS. Subsequently, $100 \mu \mathrm{L}$ of these suspensions were diluted in $900 \mathrm{~L}$ of PBS and inoculated in plates containing brain-heart infusion (BHI) agar media (Becton Dickinson $\mathrm{GmbH}$, Germany). Colonies were counted visually after $24 \mathrm{~h}$ of incubation at $37^{\circ} \mathrm{C}$ for fungal burden determination. Statistical analyses were performed using GraphPad Prism version 6.0 (GraphPad Software, San Diego, USA). Statistical comparisons were made by analysis of variance (one-way ANOVA) followed by a Tukey-Kramer post-test. p-values of $<0.05$ indicated statistical significance. Results show mean \pm standard deviation (SD) of three independent experiments.

Phylogenetic analysis of trehalases and trehalose utilisation in pathogenic/opportunistic yeasts - Phylogenetic trees were assembled with trehalases sequences from different pathogenic yeasts obtained from the Basic Local Alignment Search Tool (BLAST, https:// blast.ncbi.nlm.nih.gov/Blast.cgi) or from the Yeast Gene Order Browser (YGOB) (http://ygob.ucd.ie) or Candida Gene Order Browser $(C G O B)$ (http://cgob.ucd.ie) databases and then aligned using the Multiple Sequence Comparison by Log-Expectation tool (MUSCLE, https:// www.ebi.ac.uk/Tools/msa/muscle). The alignment file was used to generate the phylogenetic tree using Akaike Information Criterion (AIC) in Smart Model Selection implemented in the PhyML environment (http://www. atgc-montpellier.fr/phyml-sms), with a bootstrap analysis of 1,000 replicates. For the management of the phylogenetic tree, we used the online tool Interactive Tree of Life (iTOL, https://itol.embl.de). Open reading frames from non-annotated sequences of putative trehalases were obtained through BLAST against Whole Genome Shotgun (WGS) or BioProject contigs in National Center for Biotechnology Information (NCBI), and then defined using ORFfinder tool (https://www.ncbi.nlm. nih.gov/orffinder/). Protein structure and functional do- mains of the amino acid sequences were analysed using the EMBL-EBI InterPro website (http://www.ebi.ac.uk/ interpro/). The capacity of the different yeasts to assimilate (grow on) and/or ferment trehalose was verified in the Westerdijk Fungal Biodiversity Institute website (http://www.wi.knaw.nl/) using the description of type strains, as well as from a reference book. ${ }^{(15)}$

\section{RESULTS}

Deletion of the CgATH1 gene impairs growth on trehalose by C. glabrata - In chromosome K of the known genome of the C. glabrata strain CBS138 is located a gene (locus CAGLOK05137g) in synteny and with significant (68\%) identity with the gene encoding the ATH1 acid trehalase from $S$. cerevisiae. ${ }^{(16)}$ We have already shown that the expression of this $C g A T H 1$ gene in $S$. cerevisiae allows efficient trehalose consumption and fermentation. ${ }^{(12)}$ In order to gain knowledge into the in vivo function of this gene in C. glabrata, we took advantage of the promoter-dependent disruption of genes (PRODIGE) method ${ }^{(14)}$ to delete this gene from the genome of strain Bg14 (Supplementary data, Table I). This method (Fig. 1) has been successfully used to analyse the effects of deleting specific genes in the susceptibility/resistance of $C$. glabrata strains challenged with different drugs, or even during macrophage killing. ${ }^{(17,18)}$

Since we have previously shown that the acid trehalase is glucose repressed, and high levels of secreted enzyme activity were obtained when the yeast cells are grown in glycerol, ${ }^{(12)}$ the transformants were selected in minimal YNB medium plates with $30 \mathrm{~g} \mathrm{~L}^{-1}$ of glycerol as carbon source. From more than 20 colonies that showed growth on YNB-30 g L-1 glycerol plates, two of them failed to grow in YNB-20 $\mathrm{g} \mathrm{L}^{-1}$ trehalose plates and PCR analysis confirmed that $S c U R A 3$ gene was correctly integrated in chromosome $\mathrm{K}$, deleting the $\mathrm{CgATH1}$ gene in these strains (Fig. 1). One of these aht $1 \Delta$ mutant strains was used for further characterisation, and as can be seen in Fig. 2, this aht1 $\Delta$ strain could grow normally in rich YP-20 g L-1 glucose medium (Fig. 2A), but failed to grow in rich medium containing $20 \mathrm{~g} \mathrm{~L}^{-1}$ trehalose as carbon source (Fig. 2B). Same results were obtained in synthetic YNB medium with $20 \mathrm{~g} \mathrm{~L}^{-1}$ of glucose or trehalose (data not shown).

Trehalase activity in C. glabrata cells - The wildtype strain had a periplasmic acid trehalase activity of $58.3 \pm 14.2 \mathrm{mU} / \mathrm{mg}$ CDW after growth on rich YP medium with $20 \mathrm{~g} \mathrm{~L}^{-1}$ of glucose, and an activity of 131 $\pm 48 \mathrm{mU} / \mathrm{mg} \mathrm{CDW}$ when grown on the same medium containing $30 \mathrm{~g} \mathrm{~L}^{-1}$ of glycerol. The aht $1 \Delta$ mutant strain showed less than $0.3 \mathrm{mU} / \mathrm{mg} \mathrm{CDW}$ of periplasmic trehalase activity after growth on any of these carbon sources. The wild-type strain secreted more than $480 \pm$ $130 \mathrm{mU} / \mathrm{mL}$ of acid trehalase activity into the medium after growth on glucose or glycerol, but we could not detect any acid trehalase activity secreted into the medium by the aht $1 \Delta$ mutant strain.

Besides the CgATH1 gene encoding for an extracellular acid trehalase, the known genome of C. glabrata contains two other putative trehalase genes ( $C A$ GLOC04323g and CAGLOM10439g) that are syntenic 


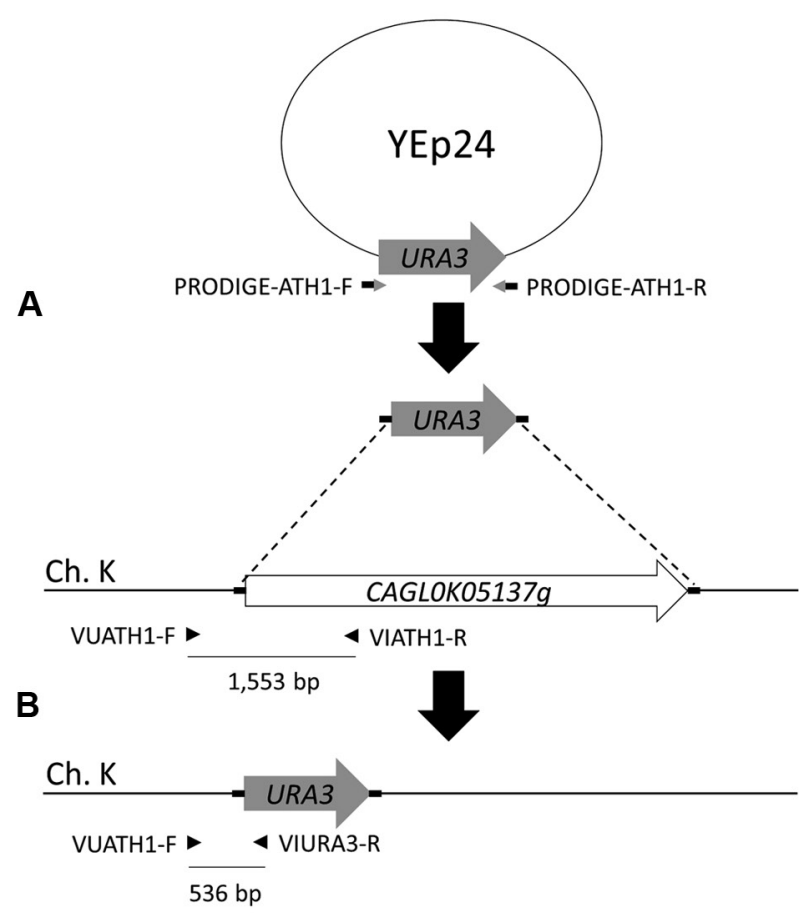

C

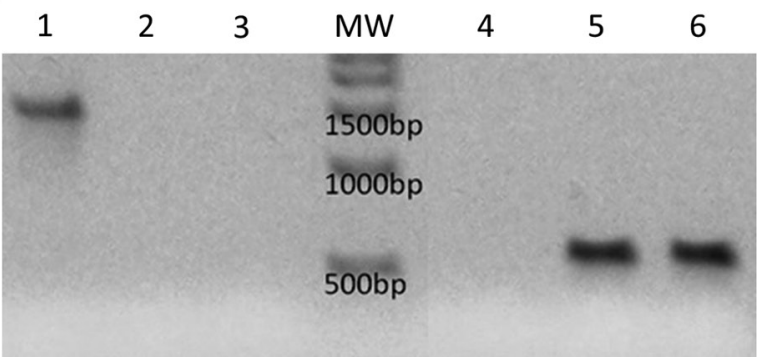

Fig. 1: deletion of the CgATH1 gene in Candida glabrata using the promoter-dependent disruption of genes (PRODIGE) method. (A) The URA3 gene present in plasmid YEp24 was amplified with two primers having homology $(\sim 60 \mathrm{nt})$ to the immediately upstream and downstream regions of the CgATH1 gene (ORF CAGLOK05137g) present in chromosome $\mathrm{K}$. After transformation and selection in minimal YNB medium, the correct replacement of the $C g A T H 1$ gene by URA3 through recombination (B) was confirmed by PCR. (C) Gel electrophoresis of the products obtained with primers VUATH1-F and VIATH1-R and the genomic DNA of the wild-type strain (Lane 1) and its absence from two aht $1 \Delta$ mutant strains (Lanes 2 and 3) or with primers VUATH1-F and VIURA3-R, which did not amplified any fragment from the wild-type strain (Lane 4), but produced the expected fragment from the two aht $1 \Delta$ mutant strains (Lanes 5 and 6 ), indicating that the ScURA3 gene was correctly integrated at the chromosome, deleting the $C g A T H 1$ gene.

with a couple of paralogs with $70 \%$ identity that arose from the WGD event, and share $67-79 \%$ identity with the NTH1 and NTH2 genes from S. cerevisiae, which encode for the intracellular neutral trehalases. ${ }^{(16)}$ However, due to the very high activity of the acid trehalase present in the wild-type C. glabrata strain, it was difficult to visualise the activity of the cytoplasmic neutral trehalase in cell extracts (Fig. 3A). However, this activity can be clearly seen in cell extracts of the aht $1 \Delta$ strain
(Fig. 3B), with an optimal pH of 6.5-7.0. It is important to note that the neutral trehalase activity in aht $1 \Delta$ yeast cells was very low, when compared to the high acidic trehalase activity present in the wild-type strain. No differences were observed in the intracellular trehalose concentration or intracellular trehalose mobilisation during growth on glucose by the wild-type or ahtld yeast strains (data not shown).

The acid trehalase encoded by CgATH1 is involved in C. glabrata virulence - The effect of deleting $\mathrm{CgATH1}$ on the virulence of $C$. glabrata was determined in a murine model of disseminated infection, both in normal or immunocompromised mice (Fig. 4). After caudal i.v. yeast inoculation in immunocompetent mice, a high number of colony-forming units (CFUs) of the C. glabrata wild-type strain were found in the mice spleen eight days after infection, but much less in the kidney (Fig. 4A). The aht $1 \Delta$ mutant strain underwent a significant loss of virulence, as shown by the lower number of CFUs found in the spleen, compared to those recorded by the wild-type strain, reaching the same low values observed when the infected mice were treated with fluconazole (Fig. 4A). Actually, treatment with fluconazole did not further reduce the fungal burden of the aht $1 \Delta$ strain. A slightly different pattern was observed in immunocompromised mice since besides the spleen, kidneys were also significantly infected (Fig. 4B), but in both tissues the fungal burden by the ahtl $\Delta$ mutant strain was significantly lower than the $C$. glabrata wild-type strain. Thus, our results indicate that extracellular trehalose hydrolysis and metabolisation are important for virulence of this fungal pathogen in the mammalian host. Considering that extracellular trehalases are known virulence factors for entomopathogenic fungus, since trehalose is the main sugar in the haemolymph of insects, we refrained from using an alternative insect infection bioassay (e.g., with Galleria mellonella larvae or silkworm) to determine the virulence of the ath $1 \Delta$ mutant strain.

Trehalases and extracellular trehalose utilisation by pathogenic/opportunistic Candida yeasts - We analysed the ability to assimilate (grow on) and ferment the disaccharide trehalose by the major pathogenic/opportunistic Candida yeast species $^{(1)}$ with assembled genome sequences available (a total of 28 species, including the opportunistic yeast $S$. cerevisiae) and performed a phylogenetic analysis of the genes encoding trehalases present in those yeasts (Fig. 5). A total of 24 Candida yeast species had genes encoding for acid trehalases (with $\geq$ $35 \%$ identity with ATH1 from S. cerevisiae (Supplementary data, Tables II, III) and all of them were able to assimilate trehalose from the medium (including "weak" or "slow/delay" phenotypes (Fig. 5A, B). More than $70 \%$ of these trehalose positive species also showed some degree of trehalose fermentation (including "positive", "variable", "slow/delay" or "weak" phenotypes).

Considering the five major pathogenic Candida yeast species (C. albicans, C. glabrata, C. krusei, C. parapsilosis and C. tropicalis), responsible for approximately $93 \%$ of all invasive candidiasis cases ${ }^{(1)}$ only C. krusei (syn. Pichia kudriavzevii) lacks a gene that encodes for an acid trehalase, and is unable to assimilate (or ferment) 


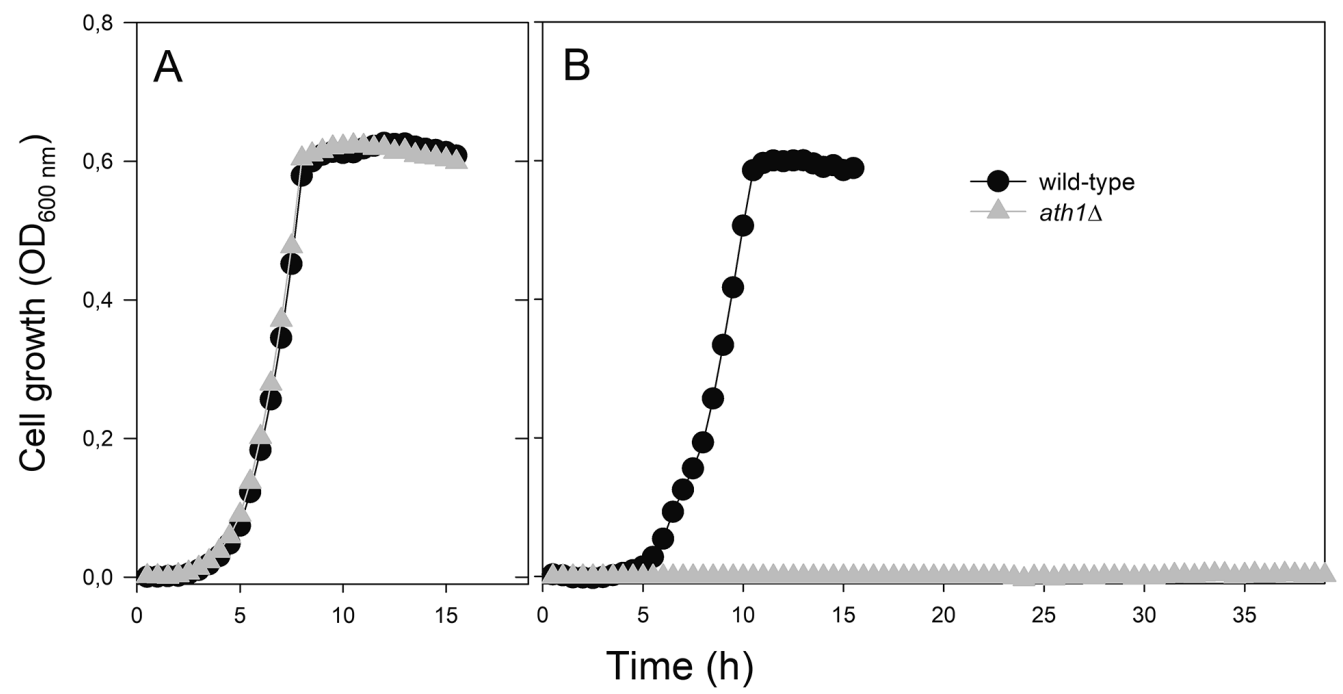

Fig. 2: growth of the Candida glabrata wild-type or aht1 $\Delta$ mutant strain in rich YP medium containing $20 \mathrm{~g} \mathrm{~L}^{-1}$ of glucose (A) or trehalose (B) as carbon source. Yeast growth at $30^{\circ} \mathrm{C}$ was monitored with a Tecan GENios microplate reader by measuring the OD ${ }_{600}$ every 30 min, with high intensity orbital shaking between measurements.

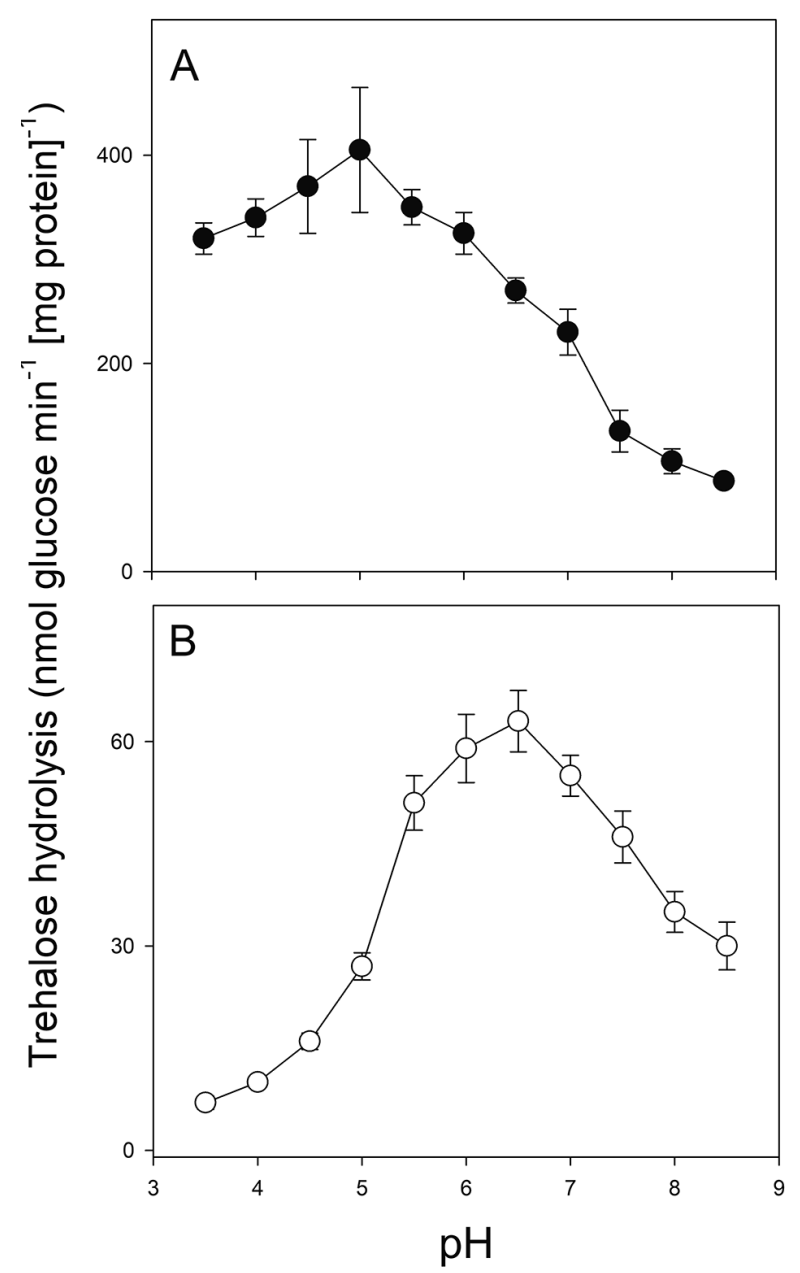

Fig. 3: trehalase activity in cell extracts of the Candida glabrata wildtype (A) or aht $1 \Delta$ mutant strain (B) determined in the indicated $\mathrm{pH}$ after growth of the cells for $24 \mathrm{~h}$ on rich YP medium containing $20 \mathrm{~g}$ $\mathrm{L}^{-1}$ of glucose. trehalose. Regarding the other 23 emerging/opportunistic pathogenic yeast species (all with less than $1 \%$ of individual incidence), 20 of them had genes encoding acid trehalases and showed growth on trehalose, of which 13 also had some degree of trehalose fermentation (Fig. 5B). We could not find genes encoding for acid trehalases in three other emerging/opportunistic Candida yeast species: C. inconspicua (syn. Pichia cactophila), C. lipolytica (syn. Yarrowia lipolytica) or C. norvegensis (syn. P. norvegensis), and as expected none of them could assimilate or ferment trehalose. It is important to note that the three pathogenic yeasts within the Nakaseomyces genus (C. glabrata, C. bracarensis and C. nivariensis $)^{(5)}$ have acid trehalases and assimilate and ferment trehalose (Fig. 5), while the three non-pathogenic species (C. castellii, N. bacillisporus and $N$. delphensis) do not consume or ferment this sugar. Overall, our analysis reveals that more than $85 \%$ of the major pathogenic and emerging/opportunistic Candida species have genes encoding for an acid trehalase and show extracellular trehalose assimilation, or even fermentation.

The acid trehalase encoded by the $C g A T H 1$ gene belongs to the glycosyl hydrolase family 65 (GH-65) of the Carbohydrate-Active enZyme database (CAZy, http:// afmb.cnrs-mrs.fr/CAZY). These enzymes are extracellular because they could present a transmembrane domain close to the $\mathrm{N}$-terminal (TM) (Fig. 5C-i) responsible for anchoring the protein at the plasma membrane and/or cell wall ${ }^{(19)}$ or even promoting the secretion of the enzyme into the medium, as in the case of $C$. glabrata. $^{(12)}$ Only five yeast species (C. auris, $C$. ciferrii, $C$. fermentati, $C$. kefyr and C. pelliculosa) have slightly shorter proteins with a signal peptide at the N-terminal end of the sequence (Fig. 5C-ii), indicating that they are also probably secreted into the medium. Only two species $(C$. haemulonii and $C$. tenuis) have proteins with $\mathrm{N}$-terminal domains with different sizes and lacking any of these targeting 

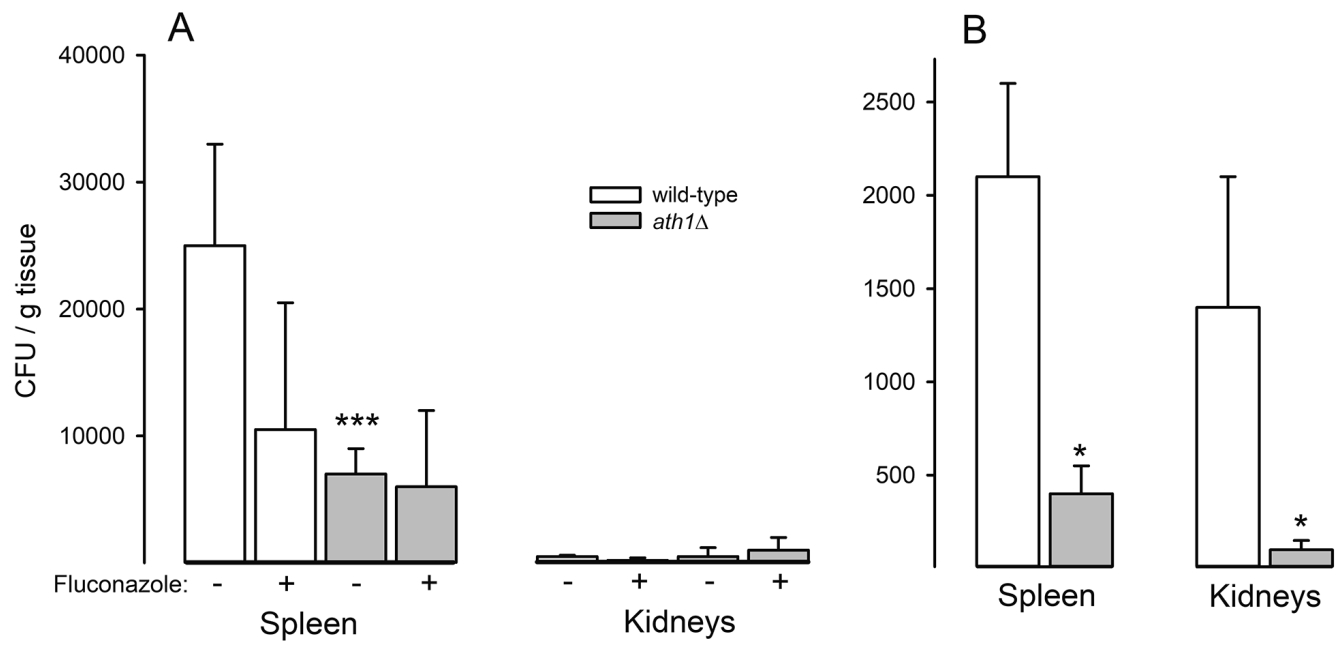

Fig. 4: fungal burden in spleens and kidneys from (A) immunocompetent BALB/c mice inoculated with $3 \times 10^{5}$ yeast cells of Candida glabrata wild-type or ath $1 \Delta$ strain and treated daily with (or without) fluconazole as indicated. (B) Cyclophosphamide-treated immunosuppressed mice inoculated with $1 \times 10^{3}$ yeast cells of $C$. glabrata wild-type or athl $\Delta$ strain. In all cases, colony-forming units (CFUs) were determined eight days post-challenge. Statistical analysis was carried out using the analysis of variance (one-way ANOVA) with post Tukey-Kramer test: * $<<$ $0.01 ; * * * \mathrm{p}<0.0001$ between the wild-type and ath $1 \Delta$ strain

signals (Fig. 5C-iii). Thus, their involvement in extracellular trehalose hydrolysis remains to be proven experimentally. It is important to note that trehalose might also be directly transported into the cell, where it can be hydrolysed by cytoplasmic neutral trehalases, as has been described in S. cerevisiae. ${ }^{(11)}$ An initial survey of putative trehalose transporters in C. haemulonii and C. tenuis revealed several genes (data not shown) with all the characteristics described for such permeases, including those amino acids required for sugar binding and transport. ${ }^{(20)}$

Indeed, we found genes encoding for neutral trehalases in all the pathogenic yeast species analysed. Supplementary data, Figure, shows the enzymes found in the 24 pathogenic yeasts of Fig. 5 plus two other yeast species that lack acid trehalase genes $(C$. krusei and $C$. incons-picua). Neutral trehalases belong to the GH family 37 (GH-37) (which includes a $\mathrm{Ca}^{2+}$ binding domain) in the CAZy database and, as mentioned before, these enzymes are responsible for intracellular hydrolysis of trehalose ${ }^{(16)}$ In $S$. cerevisiae and other yeasts that underwent the WGD, two paralogs are found: NTH1 and NTH2, but most pathogenic/opportunistic yeast species had genes encoding for a single neutral trehalase, either with higher identity to NTH1 (21 genes) or to NTH2 (seven genes). Surprisingly, C. krusei been the more distantly related species, also had two genes encoding for neutral trehalases (Supplementary data, Figure, Table II).

\section{DISCUSSION}

The disaccharide trehalose is an important sugar for several microorganisms, plants and insects. Trehalose is considered not only a storage carbohydrate and stressprotectant, but it also acts as regulator of many cellular processes. Since humans do not synthetise trehalose, the enzymes involved in trehalose metabolism by pathogenic yeasts have been studied as interesting and potentially antifungal targets for chemotherapy. ${ }^{(21)}$ Our results show that the $C$. glabrata secreted acid trehalase encoded by the CgATH1 gene is required for growth on trehalose and contributes to the virulence of the yeast. There are at least two other examples were deletion of the acid trehalase decreases infectivity by pathogenic yeasts. The acid trehalase present in C. albicans, which is required for growth on trehalose, ${ }^{(22)}$ is involved in the virulence of this major pathogenic yeast. ${ }^{(23)}$ Same results have been reported for the acid trehalase of C. parapsilosis. ${ }^{(24)}$ More recently, a trehalase required for growth on trehalose has been also implicated in virulence of the bacteria Burkholderia pseudomallei.(25)

An intriguing issue raised by these results is why these pathogenic microorganisms have the ability to consume extracellular trehalose as carbon source (Fig. 5 ), since this sugar is not normally found in the environment or mammalian host, and its implications for virulence. A possible scenario is that during colonisation and infection, when many microorganisms are being killed by the harsh environment and immune defences of the host (thus, liberating trehalose into the medium), having the ability to consume (or even ferment) trehalose could be an advantageous trait for a pathogen. Indeed, humans also expresses the enzyme trehalase as a glycoprotein in the small intestine and renal brush-border membranes, and while the trehalase in the intestine is involved in the hydrolysis of ingested trehalose, in turn, the physiological role of trehalase in the kidney has remained more elusive, but certainly could hydrolyse trehalose released by dying microorganisms present in the urinary tract. Dietary trehalose is also a concern, since recent results indicate that hypervirulent epidemic Clostridioides difficile ribotypes have enhanced capacity to consume trehalose, and this sugar increases the virulence of such strains in a murine model of infection. ${ }^{(26)}$ Unfortunately, the known trehalase competitive inhibitor validamycin A had limited antifungal effects with $C$. 


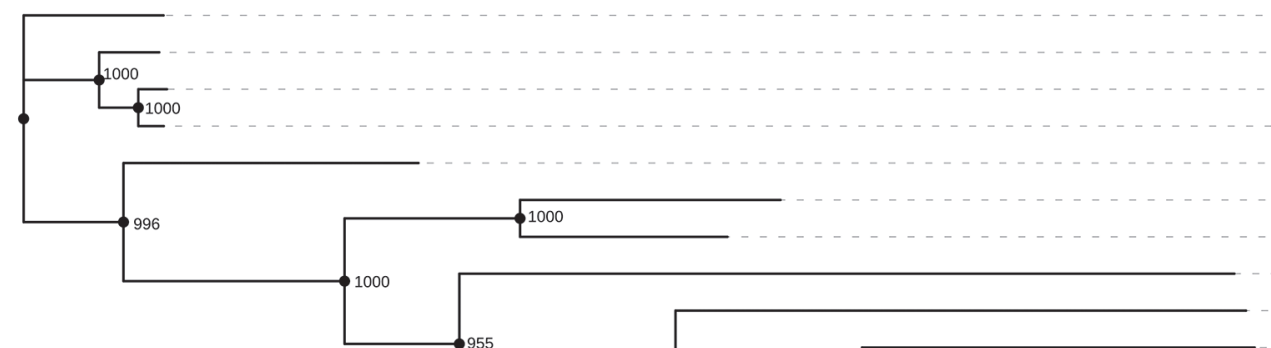

cerevisiae

C. glabrata

\section{C} i)

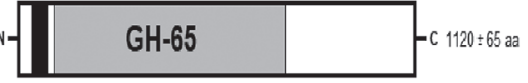

ii)

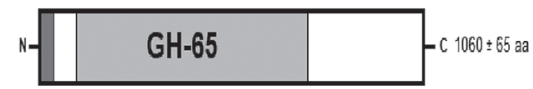

iii)

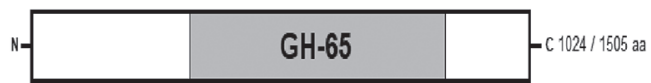

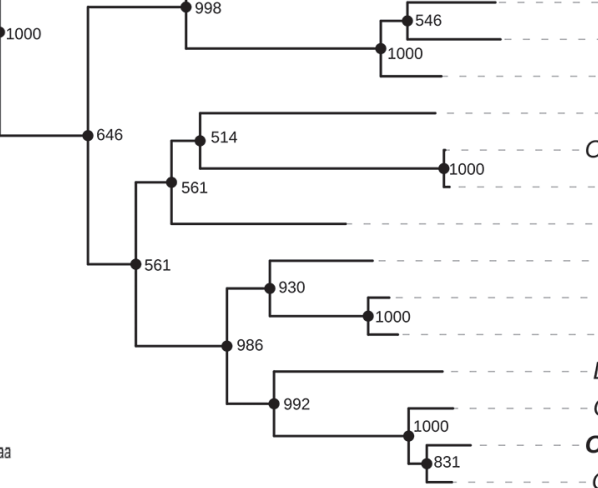

- C. tenuis

C. quilliermondii $+\quad v$

C. fermentati

C. tropicalis ++

C. albicans + v/w/S

C. dubliniensis $+\quad-$

L. elongisporus + +

C. metapsilosis $+\mathrm{v}$

C. parapsilosis $+\quad \mathrm{v}$

C. orthopsilosis $+\mathrm{v}$

Fig. 5: acid trehalases and trehalose assimilation and fermentation by pathogenic/opportunistic Candida species. (A) Phylogenetic tree of characterised (bold) and other predicted acid trehalases present in the indicated yeast species. The numbers next to the nodes represent the bootstraps values (based on 1,000 replications). (B) Data regarding extracellular trehalose assimilation (Ass.) or fermentation (Fer.) by the yeasts -+: positive; -: negative; w: weak; s: slow/delayed; v: variable. (C) Schematic representation of the acid trehalases and their functional domain content, including the glycosyl hydrolase family 65 (GH-65) trehalase domain (grey), the TM segment (black, i), a signal peptide (dark grey, ii) or even absence of these last two domains in some predicted enzymes (iii).

albicans. ${ }^{(27)}$ However, a recent publication showed that validamycin A had a synergistic inhibitory effect with amphotericin B against the pathogenic fungus Aspergillus flavus, without cytotoxic effects in human bronchial epithelial cells, indicating its possible use in vivo for treatment of fungal infections. ${ }^{(28)}$ Nevertheless, new trehalose analogues, including those designed to resist enzymatic degradation that inhibit trehalose utilisation by hypervirulent $C$. difficile ${ }^{(29)}$ may constitute new clinically interesting antifungal-related compounds. Thus, more studies are required to elucidate the role of extracellular trehalose and the high levels of secreted acid trehalase has in the physiopathology of C. glabrata and other yeast pathogens (Fig. 5).

Our analysis of both experimentally characterised and predicted trehalases in the major pathogenic/opportunistic Candida yeast species (Fig. 5 and Supplementary data, Figure) revealed that acid trehalases have the classical GH-65 domain and either a TM $(70 \%$ of the acid trehalases) (Fig. 5C-i) or signal peptide (21\%) (Fig. $5 \mathrm{C}$-ii). These sequences allow extracellular localisation, or even secretion, of the enzyme into the medium, as already described for known acid trehalases of yeast and other fungi. ${ }^{(19)}$ The only exceptions were the predicted acid trehalases found in $C$. haemulonii and $C$. tenuis, lacking any of these targeting domains (Fig. 5C-iii), and thus the mode of extracellular trehalose assimilation by these yeasts needs further studies. Regarding the neutral trehalases, we found a more diverse scenario regarding this enzyme in the analysed pathogenic/opportunistic yeast, with the majority of species having a single enzyme with the GH-37 domain (either of the NHT1 or NHT2 type of neutral trehalases), while some yeast species that underwent the WGD had both paralogs (A and B-i in Supplementary data, Figure). Indeed, within the WGD yeast species, some have a single gene homologous to NTH1 (e.g., Kazachstania naganishii), others a single gene homologous to NTH2 (e.g., K. africana, Naumovozyma castellii and $N$. diarenensis), while other yeast species have genes homologous to the two neutral trehalases (e.g., Tetrapisispora blattae, T. phaffii and Vanderwaltozyma polyspora).

It is also significant to note that the intracellular neutral trehalase activity in C. glabrata is only clearly detected in the ath $1 \Delta$ mutant strain (Fig. 3), and since this enzyme in $S$. cerevisiae is a known target of the cAMP/ PKA nutrient signalling pathway, promoting the switch between respirative/gluconeogenic growth and fermentative growth, ${ }^{(30)}$ the mutant ath $1 \Delta$ strain is a promising platform to characterise the C. glabrata orthologues of the known receptors and transceptors (transporter receptors) involved in nutrient sensing and signalling through the cAMP/PKA signalling pathway. In fungal pathogens, the sensing of sugars is important for a number of virulence attributes, including adhesion, oxidative stress resistance, biofilm formation, morphogenesis, invasion 
and antifungal drug tolerance, thus the understanding of sugar sensing and metabolism may offer new valuable antifungal drug targets. ${ }^{(9)}$

In conclusion, in C. glabrata, the deletion of the CgATH1 gene encoding for the secreted acid trehalase impairs the use of this carbon source for growth, reveals the activity of the intracellular neutral trehalase, and reduces the fungal burden in a murine model of infection, both in normal or immunocompromised mice.

\section{ACKNOWLEDGEMENTS}

To Dr BP Cormack (Johns Hopkins University School of Medicine, Baltimore, USA), for supplying strains $\mathrm{Bg} 2$ and $\mathrm{Bg} 14$. The funding sources were not involved in the study design, analysis and interpretation of data, or in the decision to submit the article for publication.

\section{AUTHORS' CONTRIBUTION}

RGL, JEM and LMB designed and performed experiments. SLA-Jr analysed data and contributed new methods. CPT and BUS participated in designing the study, contributed new models, and provided financial support. BUS wrote the manuscript, which was revised and approved by all authors. The authors declare no conflict of interests.

\section{REFERENCES}

1. Pfaller MA, Diekema DJ, Turnidge JD, Castanheira M, Jones RN. Twenty years of the SENTRY antifungal surveillance program: Results for Candida species from 1997-2016. Open Forum Infect Dis. 2019;6(Suppl 1):S79-94.

2. Colombo AL, Garnica M, Aranha Camargo LF, Da Cunha CA, Bandeira AC, Borghi D, et al. Candida glabrata: an emerging pathogen in Brazilian tertiary care hospitals. Med Mycol. 2013;51(1):38-44.

3. Mario DA, Denardi LB, Bandeira LA, Antunes MS, Santurio JM, Severo LC, et al. The activity of echinocandins, amphotericin $\mathrm{B}$ and voriconazole against fluconazole-susceptible and fluconazole-resistant Brazilian Candida glabrata isolates. Mem Inst Oswaldo Cruz. 2012;107(3):433-6.

4. Galocha M, Pais P, Cavalheiro M, Pereira D, Viana R, Teixeira MC. Divergent approaches to virulence in C. albicans and C. glabrata: two sides of the same coin. Int J Mol Sci. 2019;20(9):e2345.

5. Gabaldón T, Martin T, Marcet-Houben M, Durrens P, BolotinFukuhara M, Lespinet O, et al. Comparative genomics of emerging pathogens in the Candida glabrata clade. BMC Genomics. 2013;14:623.

6. Brown CA, Murray AW, Verstrepen KJ. Rapid expansion and functional divergence of subtelomeric gene families in yeasts. Curr Biol. 2010;20(10):895-903.

7. Domergue R, Castano I, De las Penas A, Zupancic M, Lockatell $\mathrm{V}$, Hebel JR, et al. Nicotinic acid limitation regulates silencing of Candida adhesins during UTI. Science. 2005;308(5723):866-70.

8. Iosue CL, Attanasio N, Shaik NF, Neal EM, Leone SG, Cali BJ, et al. Partial decay of thiamine signal transduction pathway alters growth properties of Candida glabrata. PLoS One. 2016;11(3):e0152042.

9. Van Ende M, Wijnants S, Van Dijck P. Sugar sensing and signaling in Candida albicans and Candida glabrata. Front Microbiol. 2019;10:99.
10. Fraser M, Borman AM, Johnson EM. Evaluation of the commercial rapid trehalose test (GLABRATA RTT) for the point of isolation identification of Candida glabrata isolates in primary cultures. Mycopathologia. 2012;173(4):259-64.

11. Jules M, Guillou V, François J, Parrou J. Two distinct pathways for trehalose assimilation in the yeast Saccharomyces cerevisiae. Appl Environ Microbiol. 2004;70(5):2771-8.

12. Zilli DM, Lopes RG, Alves SL Jr, Barros LM, Miletti LC, Stambuk BU. Secretion of the acid trehalase encoded by the $C g A T H 1$ gene allows trehalose fermentation by Candida glabrata. Microbiol Res. 2015;179:12-9.

13. Rasheed M, Kumar N, Kaur R. Global secretome characterization of the pathogenic yeast Candida glabrata. J Proteome Res. 2020;19(1):49-63.

14. Edlind TD, Henry KW, Vermitsky JP, Edlind MP, Raj S, Katiyar SK. Promoter-dependent disruption of genes: simple, rapid, and specific PCR-based method with application to three different yeast. Curr Genet. 2005;48(2):117-25.

15. Kurtzman CP, Fell JW, Boekhout T. The Yeasts: a taxonomic study. 5th ed. Netherland: Elsevier; 2011.

16. Nwaka S, Holzer H. Molecular biology of trehalose and the trehalases in the yeast Saccharomyces cerevisiae. Prog Nucleic Acid Res Mol Biol. 1998;58:197-237.

17. Nevitt T, Thiele DJ. Host iron withholding demands siderophore utilization for Candida glabrata to survive macrophage killing. PLoS Pathog. 2011;7(3):e1001322.

18. Katiyar SK, Alastruey-Izquierdo A, Healey KR, Johnson ME, Perlin DS, Edlind TD. Fks1 and Fks2 are functionally redundant but differentially regulated in Candida glabrata: implications for echinocandin resistance. Antimicrob Agents Chemother. 2012;56(12):6304-9.

19. Parrou JL, Jules M, Beltran G, François J. Acid trehalase in yeasts and filamentous fungi: localization, regulation and physiological function. FEMS Yeast Res. 2005;5(6-7):503-11.

20. Trichez D, Knychala MM, Figueiredo CM, Alves SL Jr, da Silva MA, Miletti LC, et al. Key amino acid residues of the $A G T 1$ permease required for maltotriose consumption and fermentation by Saccharomyces cerevisiae. J Appl Microbiol. 2019;126(2):580-94.

21. Perfect JR, Tenor JL, Miao Y, Brennan RG. Trehalose pathway as an antifungal target. Virulence. 2017;8(2):143-9.

22. Pedreno Y, Maicas S, Arguelles JC, Sentandreu R, Valentin E. The $A T C 1$ gene encodes a cell wall-linked acid trehalase required for growth on trehalose in Candida albicans. J Biol Chem. 2004;279(39):40852-60.

23. Pedreno Y, Gonzalez-Parraga P, Martinez-Esparza M, Sentandreu R, Valentin E, Arguelles JC. Disruption of the Candida albicans ATCl gene encoding a cell-linked acid trehalase decreases hypha formation and infectivity without affecting resistance to oxidative stress. Microbiology. 2007;153(5):1372-81.

24. Sánchez-Fresneda R, Martínez-Esparza M, Maicas S, Argüelles JC, Valentín E. In Candida parapsilosis the ATC1 gene encodes for an acid trehalase involved in trehalose hydrolysis, stress resistance and virulence. PLoS One. 2014;9(6):e99113.

25. Vanaporn M, Sarkar-Tyson M, Kovacs-Simon A, Ireland PM, Pumirat P, Korbsrisate S, et al. Trehalase plays a role in macrophage colonization and virulence of Burkholderia pseudomallei in insect and mammalian hosts. Virulence. 2017;8(1):30-40.

26. Collins J, Robinson C, Danhof H, Knetsch CW, van Leeuwen HC, Lawley TD, et al. Dietary trehalose enhances virulence of epidemic Clostridium difficile. Nature. 2018;553(7688):291-4. 
27. Guirao-Abad JP, Sánchez-Fresneda R, Valentín E, MartínezEsparza M, Argüelles JC. Analysis of validamycin as a potential antifungal compound against Candida albicans. Int Microbiol. 2013;16(4):217-25.

28. Plabutong N, Ekronarongchai S, Niwetbowornchai N, Edwards SW, Virakul S, Chiewchengchol D, et al. The inhibitory effect of validamycin A on Aspergillus flavus. Int J Microbiol. 2020;2020:3972415

29. Danielson ND, Collins J, Stothard AI, Dong QQ, Kalera K, Woodruff PJ, et al. Degradation-resistant trehalose analogues block utilization of trehalose by hypervirulent Clostridioides difficile. Chem Commun. 2019;55(34):5009-12.

30. Conrad M, Schothorst J, Kankipati HN, Van Zeebroeck G, Ru-
bio-Texeira M, Thevelein JM. Nutrient sensing and signaling in the yeast Saccharomyces cerevisiae. FEMS Microbiol Rev. 2014;38(2):254-99.

31. Fidel PL, Cutright JL, Tait L, Sobel JD. A murine model of Candida glabrata vaginitis. J Infect Dis. 1996;173(2):425-31.

32. Cormack BP, Falkow S. Efficient homologous and illegitimate recombination in the opportunistic yeast pathogen Candida glabrata. Genetics. 1999;151(3):979-87.

33. Navarro-Muñoz JC, de Jong AW, van den Ende BG, Hass P-J, Then ER, Tap RM, et al. The high-quality complete genome sequence of the opportunistic fungal pathogen Candida vulturna CBS 14366 ${ }^{\mathrm{T}}$. Mycopathologia. 2019;184(6):731-4. 